\title{
Variations of the Fechner wheel
}

\author{
ALLEN H. WOLACH \\ Illinois Institute of Technology, Chicago, Illinois \\ and \\ MAUREEN A. MCHALE \\ Northwestern State University of Louisiana, Natchitoches, Louisiana
}

\begin{abstract}
The Fechner wheel is a disk that is printed in black and white. When the Fechner wheel spins at an appropriate speed, semicircles on the wheel appear to be in color. Modern computer technology makes it possible to rapidly and repeatedly present a series of images on a monitor screen. A series of still images of a Fechner wheel can be substituted for a Fechner wheel on a motor shaft. Straight lines and lines of text can be substituted for semicircles to obtain a color effect. A color effect can be obtained in small areas of black-and-white pictures.
\end{abstract}

Gustav Theodore Fechner (1801-1887) started his career as a professor of physics. Fechner's main interest throughout his career as a physicist was philosophy, and his major empirical contributions were to psychology (Boring, 1950). Fechner is usually associated with psychophysics, an attempt to solve the mind-body problem in a way that would satisfy materialistic scientists of his day (Hergenhahn, 2005).

Fechner's work on color vision led him to create the Fechner wheel (Sweeney, 1998), which is sometimes referred to as Benham's top or the visual perception disk. The Fechner wheel is a disk that is printed in black and white. When the wheel spins at an appropriate speed, semicircles on the wheel appear to be in color.

Since the Fechner wheel is not printed in color, color receptors in the cones of the retina are not stimulated in the ordinary way. That is, the perceived color does not come from what early psychologists referred to as an "adequate" stimulus (Boring, 1950; Hergenhahn, 2005). The frequency at which the sectors of the Fechner wheel rotate is responsible for the color effect. The mechanism by which the Fechner wheel stimulates the photosensitive receptors of the retina is not known.

\section{Two Versions of the Fechner Wheel}

The two most common versions of the Fechner wheel are shown in Figure 1. Both versions of the Fechner wheel are half black - that is, a $180^{\circ}$ sector of the wheel is black. The remainder of the wheel is divided into sectors with semicircular lines. There are three semicircular lines in each sector. The first sector at the top left of both Fechner wheels in Figure 1 has the largest average radius for the three semicircles. Progressive sectors have smaller aver-

Correspondence concerning this article should be addressed to A. H. Wolach, Institute of Psychology, Illinois Institute of Technology, Chicago, IL 60616-3793 (e-mail: wolach@iit.edu). age radii for the semicircles. The radii for the smallest and largest semicircles in a sector are an equal distance from the radius for the middle semicircle in the sector. The distance between the radius of the smallest semicircle of a sector and the largest semicircle of the next sector is slightly greater than the distance between semicircles within a sector. The two versions of the Fechner wheel differ in that Version 1 has three sectors with semicircles, and Version 2 has four sectors with semicircles. The semicircles are drawn with thicker lines in Version 2 than in Version 1.

\section{A Conventional Fechner Wheel}

A Fechner wheel is usually made to spin at the end of a motor shaft. Alternatively, a slightly larger hole is made in the center of the Fechner wheel and a finger is used to spin the wheel on a pencil. Version 1 of the Fechner wheel produces what appear to be semicircles of red, green, and blue. Version 2 produces what appear to be semicircles of brown and dark blue. Version 2 of the Fechner wheel produces what appears to be a stronger color effect.

Demonstrations presented in this article use Version 2 of the Fechner wheel. The semicircular lines in Version 1 are not thick enough for good reproduction on most monitors. In addition, the graphic capabilities of many microcomputers are not sufficient to reproduce the spacing of the small, thin curved lines of Version 1.

The motor that turns a Fechner wheel usually turns at four revolutions per second. Motor speeds that are much slower than four revolutions per second produce no color effect. As speed increases beyond four revolutions per second, the color effect gradually dissipates. Sweeney (1998) has provided a modern circuit for controlling the speed of a motor that is used to turn the Fechner wheel.

\section{A Computer Version of the Fechner Wheel}

In recent years, computer technology has made it possible to present images to a computer monitor in rapid 


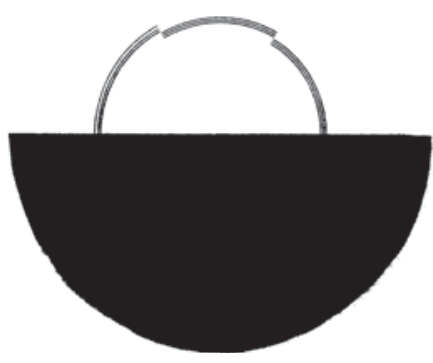

Version 1

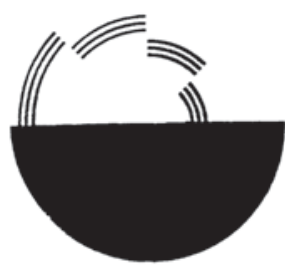

Version 2
Figure 1. Two versions of the Fechner wheel.

succession. A computer program can be used to investigate what is required to produce a color effect with blackand-white stimuli. Since the computer program can present a series of images, the images do not have to rotate on a disk attached to a mechanical system. Requisites for color effects from black-and-white images that cannot be investigated with the mechanical Fechner wheel can be investigated with a computer program.

A computer program was created to show the color effects described in this article. One part of the program can display 64 consecutive images (pictures) of Version 2 of the Fechner wheel. This portion of the program can be accessed from the Main Menu of the program.

After starting the program, the user can view the images one at a time. Successive images are rotated clockwise by $5.625^{\circ}$ from the previous image. Figure 2 shows some of the 64 consecutive images of the Fechner wheel; these images are stored in 64 bitmap (.bmp) files. (The 64 images were later edited to remove any white spaces that appeared in parts of the wheel that should be black.)

After viewing the images, the user can select "Rotate 64 Consecutive Images of the Fechner Wheel" to rapidly show the images in order. The user can enter the time in milliseconds for each image. If 1 is entered, images of the Fechner wheel are changed once every millisecond. Note that slower microcomputers cannot always show images at 1 image per millisecond. If a time is selected that is too fast for a given computer, the computer will show the images as rapidly as possible. Virtually all modern microcomputers can show the images at a speed of $20 \mathrm{msec}$ per image.

A text box labeled "Number of Images (up to 64)" is used to determine the images that are shown. If 64 is entered, all 64 images are shown consecutively. If 4 is entered, only 4 images are shown: Image 1, Image 17, Image 33, and Image 49. These 4 images are repeatedly shown in consecutive order. If 8 is entered, 8 images are shown: Image 1, Image 9, Image 17, Image 25, Image 33, Image 41, Image 49, and Image 57. These 8 images are repeatedly shown in consecutive order.

The program allows the user to choose "Constant Time for Each Image" or "Varied Time for Each Image" for stimulus presentations. If "Constant Time for Each
Image" is selected, each image is presented for the amount of time entered in the text box for "Time for Each Image in Milliseconds." If the user clicks "Varied Time for Each Image," he or she can enter a range in the text box "Range in Milliseconds." The time for successive image presentations varies within this range.

Suppose 35 is selected for "Time for Each Image in Milliseconds" and 20 is selected for "Range in Milliseconds." A quasi random number generator in the program is used to present a given image for a time between 25 and $45 \mathrm{msec}$. That is, times between 25 and $45 \mathrm{msec}$ average $35 \mathrm{msec}$, with a range of 20 .

Suppose $5 \mathrm{msec}$ is selected for the time for each image and $20 \mathrm{msec}$ is selected for the range. The quasi random number generator generates a time between -5 and $15 \mathrm{msec}$. Whenever the random number generator produces a time that is less than $1 \mathrm{msec}(\mathrm{e} . \mathrm{g} .,-2)$, the time for the image presentation is changed to $1 \mathrm{msec}$.

The program user can choose "Clockwise" to have the images appear from lowest numbered image to highest numbered image. The images are repeatedly shown in this order. When "Clockwise" is chosen, the Fechner wheel appears to rotate clockwise. "Counter Clockwise" repeatedly presents the images from highest numbered image to lowest numbered image. When "Counter Clockwise" is chosen, the Fechner wheel appears to rotate counterclockwise.

Suppose the user chooses 64 for "Number of Images," $20 \mathrm{msec}$ for "Time for Each Image in Milliseconds," "Constant Time for Each Image," and "Clockwise." The Fechner wheel appears to rotate slowly-so slowly that it is not surprising that no color effect appears on the screen. That is, the wheel appears to rotate at a speed far less than four revolutions per second, the speed required to see colors on a mechanical Fechner wheel.

Suppose the user chooses 8 for "Number of Images," 20 for "Time for Each Image in Milliseconds," and "Constant

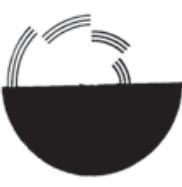

Image 1

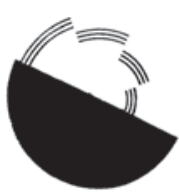

Image 5

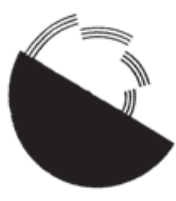

Image 9

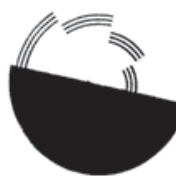

Image 2

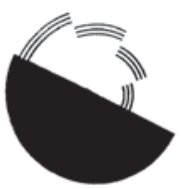

Image 6

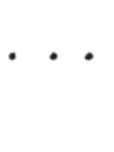


Time for Each Image." Since every eighth image is shown, the Fechner wheel appears to rotate rapidly. Colors that are comparable to the colors that would appear for the Fechner wheel attached to a motor appear on the monitor screen. Of course, the demonstration is more dramatic on a true black-and-white (monochrome) computer monitor.

A Fechner wheel must be presented in black and white to produce a color effect. The curved lines and black half of the wheel must be printed in black as opposed to a color. Most monochrome monitors are not black-and-white monitors. Monochrome cathode ray tube (CRT) monitors usually display in amber and white (or dark amber and light amber). Monochrome laptop monitors usually have blue and white (or medium blue and light blue) displays. True black-and-white monitors can be used to demonstrate the Fechner wheel. Almost all color monitors - CRT or liquid crystal display (LCD) - can produce true black-and-white images for Fechner wheel demonstrations.

CRT and LCD monitors are equally effective for presenting the Fechner wheel. Under ordinary circumstances, CRT monitors and LCD monitors produce images that do not appear to flicker, because the screen is refreshed at a rate beyond the critical flicker fusion frequency for an image that does not flicker. The speed of most mechanical versions of the Fechner wheel is four revolutions per second. The flickering that appears when a mechanical or computer-based Fechner wheel is viewed occurs because of the relatively slow speed of rotation. The refresh rate for a CRT is much faster than the rotation speed or time for image presentation for a computer-based Fechner wheel. The Fechner wheel-mechanical or computer basedappears to flicker because of the relatively long time for sector or image presentation. The preceding explanation should clarify why refresh rate does not prevent successful viewing of a Fechner wheel on a monitor screen. It should be noted that a rotating mechanical Fechner wheel can be videotaped. That is, the color effect will occur when the videotape is presented on a monitor that can produce true black-and-white images.

The computer program for the Fechner wheel demonstrates that a series of still pictures on a computer screen can be substituted for a rotating Fechner wheel on a motor shaft. As the number of images is reduced from eight to one, a number of observations can be made. No color effect occurs when one or two images are shown. A color effect occurs with three or more images (Image 1, Image 22, and Image 43). Color saturation appears to be comparable for three to eight images.

When a small number of images is used, an easy-tosee changing set of black sectors appears behind the color semicircles on the screen. For example, when three images are shown, the black sectors for an image appear in only three places on the screen. The black portions of the screen appear to flash on and off behind what appear to be colored semicircles. As the number of images increases from three to eight, the Fechner wheel loses some of the flashing black sector effect.

As the number of images increases beyond 11, the Fechner wheel at a fast speed (e.g., 1 image per $20 \mathrm{msec}$ ) appears to slow sufficiently to reduce the color effect. As the number of images continues to increase, the color effect dissipates.

When "Varied Time for Each Image" is selected, increasing the range of times for image presentations up to $30 \mathrm{msec}$ has little impact on the color effect that appears on the screen. A high number of milliseconds for "Varied Time" is analogous to an erratic motor on a mechanical system demonstrating the Fechner wheel. Apparently, constancy of rotating speed (within limits) is not necessary to produce a good color effect. When time for images varies, some images appear for a much shorter time than other images. The images that switch rapidly enough to produce image times that produce a movement effect that is analogous to four revolutions per second are sufficient to produce a color effect.

Switching from option button "Clockwise" to option button "Counter Clockwise" causes the brown and blue semicircles to reverse color. That is, when "Clockwise" is selected, the semicircles with smaller radii appear to be brown and the semicircles with larger radii appear to be blue. When "Counter Clockwise" is selected, the semicircles with smaller radii appear to be blue and the semicircles with larger radii appear to be brown.

The demonstrations described below allow the user to select the "Time for Each Image in Milliseconds." The user can also choose "Clockwise" or "Counter Clockwise" for demonstrations that involve images that are part of a circle. Similarly, the user can choose "Forward" or "Backward" for images that are not part of a circle. Switching from "Clockwise" to "Counter Clockwise" (or "Forward" to "Backward") reverses what appears to be brown and what appears to be blue in all the demonstrations.

\section{Viewing Part (One Quadrant) of the Fechner Wheel}

Mechanical system. Suppose that one has a mechanical system for displaying the Fechner wheel. A mask can be cut out of paper and placed in front of the wheel so that all but the upper right-hand quadrant of the wheel is obscured. Figure 3A shows the Fechner wheel (not rotating). Figure $3 \mathrm{~B}$ shows the mask, and Figure $3 \mathrm{C}$ shows the mask positioned in front of the Fechner wheel. When the Fechner wheel starts to spin, one obtains as strong a color effect in the visible portion of the Fechner wheel as is obtained with a completely unobscured color wheel.

When a mechanical system is used, and a mask that has an opening that is smaller than the opening described above is moved around the front of the Fechner wheel, a color effect can be seen in the portion of the Fechner wheel that is visible.

Computer presentation of one quadrant. Figure 4A shows one quadrant of each of the four images (Image 1, Image 17, Image 33, and Image 49) used with the Fechner wheel program described above (assuming the user chooses four images). Suppose the user chooses "Show 4 Images With 3 Quadrants Masked" from the Main Menu of the program. The program repeatedly presents the four images in Figure 4A in consecutive order. If the user 


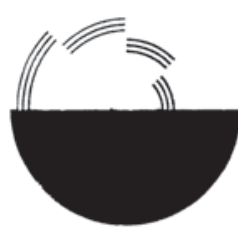

A

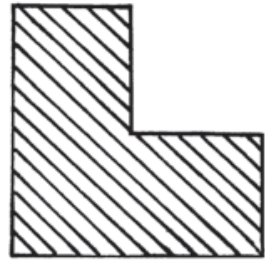

B

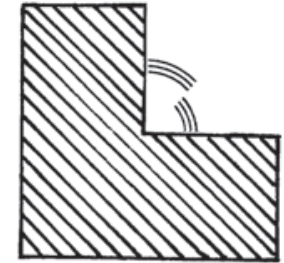

C
Figure 3. (A) The Fechner wheel, (B) the mask that is placed in front of the Fechner wheel, and (C) the Fechner wheel with the mask in front of the wheel.

chooses "Clockwise," the images are repeatedly shown in the order Image 1, Image 17, Image 33, and Image 49. If the user chooses "Counter Clockwise," the images are repeatedly shown in the order Image 49, Image 33, Image 17, and Image 1. A strong color effect appears on the screen for both clockwise and counterclockwise presentations. The preceding computer demonstration shows that images of the Fechner wheel do not have to include the complete wheel in order to produce a color effect. In the present example, only the upper right-hand quadrant of the Fechner wheel is presented for each image.

\section{Color Effects That Cannot Be Demonstrated With a Mechanical System}

Removing half of the black portion of the Fechner Wheel. Figure 4B shows the first three images that are shown in Figure 4A. One of the black quadrants has been removed from the sequence of images. A strong color effect is obtained. The black background time can be reduced by one half without disrupting the color effect.

If the order of Images 1 and 2 in Figure 4B (or Figure 4A) is reversed, the semicircles that appear to be brown and the semicircles that appear to be blue are reversed. Color for a set of semicircles is determined by the order in which the images are presented, not by the location of the semicircles on a sector. It is possible to make a Fechner wheel for a mechanical system in which the diameters for semicircles do not decrease for successive sectors of the wheel. For example, the first sector can have a smaller average diameter for the semicircles than the second sector. The order in which the sectors are placed, not the diameter of the semicircles in a sector, determines the color of the semicircles in a given sector.

Sectors with semicircles must be contiguous. That is, black sectors cannot be placed within the sectors with semicircles. For example, the images in Figure 4B will produce a color effect in the order Image 1, Image 2, Image 3, or in the order Image 2, Image 1, Image 3, but not in the order Image 1, Image 3, Image 2, or Image 2, Image 3, Image 1.

Transition from wheel to square images. Suppose the user chooses "Show 3 Images With 3 Quadrants Masked, Square Black Background.” The three images in Figure $4 \mathrm{C}$ are repeatedly presented in consecutive order.
A strong color effect appears on the screen. The black background in the last image does not have to be in part of a circle to produce a color effect. That is, the black background can cover the entire frame for the black image.

Replacing semicircles with straight lines. The computer program repeatedly presents the three images in Figure 4D in consecutive order. The user can enter the number of milliseconds for each image presentation. Note that the straight lines in the first two panels of Figure 4D start at the same height at which the curved lines start in the corresponding panels of Figures 4A, 4B, and 4C. A strong color effect appears on the screen. The six lines in each of the first two panels of Figure 4D do not have to be curved in order to produce a color effect.

Substituting printed words for some straight lines. The computer program repeatedly presents the three images in Figure 4E in consecutive order. The user can enter the number of milliseconds for each image presentation. A strong color effect appears on the screen, and lines with words show the words in the same color as the line of text would be if a straight line were substituted for the line of text. The height of the letters in the text approximates the height of the individual straight lines used in this example and the previous example.

Removing all straight lines and substituting large text. The computer program repeatedly presents the three images (Figure 4F) in consecutive order. The words are in large (14-point) font. The words are not at the same height as in the previous examples, and the lines of text are not positioned in relation to the positioning of straight lines in previous examples. The number of lines of text does not match the number of lines in previous examples with lines grouped in sets of three. A strong color effect appears on the screen. When "Forward" is selected, the words "To be or not" and "that is the" appear in brown. The words "to be" and "the question" appear in blue. When "Backward" is selected, the words "To be or not" and "that is the" appear in blue. The words "to be" and "the question" appear in brown.

When "Forward" is selected and three images are shown, words in the first of the two images before the black square appear in brown. The words in the second of the two images before the black square appear in blue. Font size and placement of lines of text on an image are not particularly critical to producing a color effect. If the order of the two images with text (the first two images) is reversed (Image 2, Image 1, Image 3), the colors of the lines of text (brown and blue) are reversed. As in other examples, the order in which images are presented determines the color of the lines of text on the image: brown text for words or lines on the first image, and blue text for words or lines on the second image.

Note that the images are presented in the order image with text (Image 1), image with text (Image 2), image with black square (Image 3). The images will not produce color if they are repeatedly presented in the order image with text (Image 1), image with black square (Image 3), image with text (Image 2). That is, the black square cannot 
A

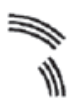

B

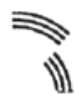

C

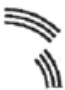

D

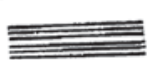

$\mathbf{E}$

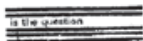

F

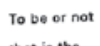

that is the

G
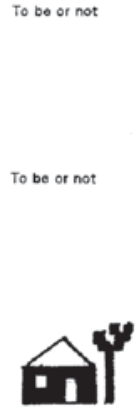

Image 1

$\mathbf{H}$

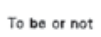

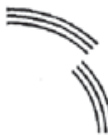
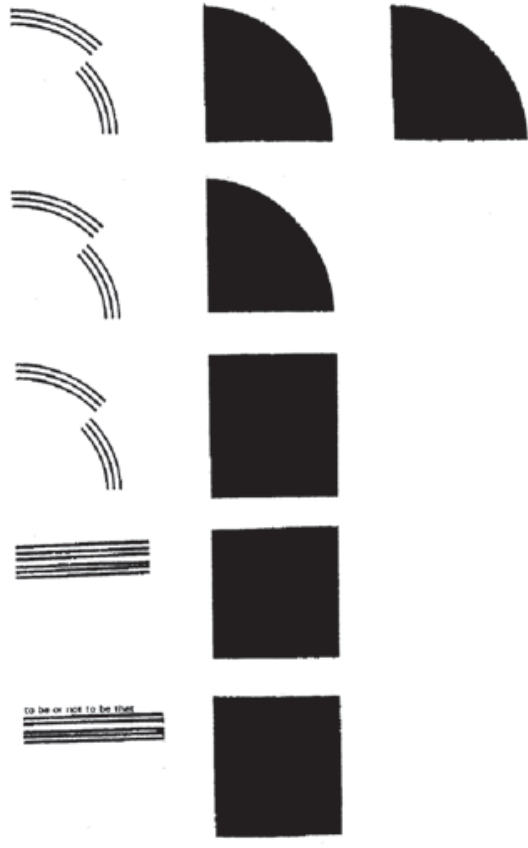

to be

the question

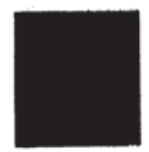

to be

That is the
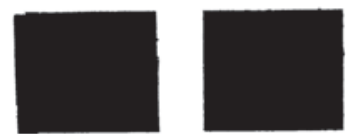

that is the
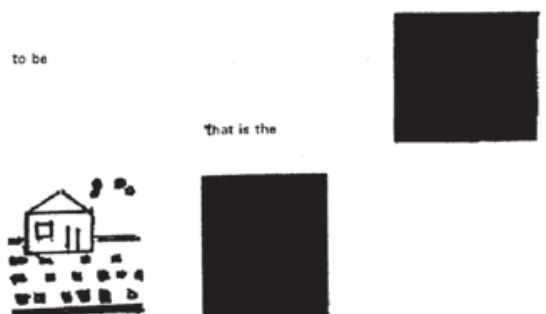

Image 2

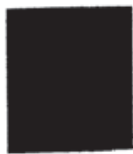

Image 3
Image 5

Figure 4. Images for each of the nine demonstrations (A-I) described in the text. For example, the first demonstration (A) uses the four images in the top row.

be between the two images with text (or lines). If the same image with text is used for both text images (e.g., image with text [Image 1], image with text [same as Image 1], image with black square [Image 3]), the text appears in black, not in the additive mixture of brown and blue.

Introducing a color between brown and blue. The first three images in Figure $4 \mathrm{G}$ have words, and the last two images are black. The words in the first image appear in brown, the words in the second image appear in medium blue, and the words in the third image appear in dark blue.
Decreasing black time when three text images are shown. Figure $4 \mathrm{H}$ shows the first four of the five images in Figure 4G. The colors that are produced are essentially the same as the colors that are produced in the previous example with five images. The amount of time for which the image is black can be reduced by one half without disrupting the color effect. In fact, a color effect can be obtained when only one black image follows the three images with words. That is, the amount of time that the screen is black can be one third the total time for the three images with words. 
Strong color effects can be obtained when 14-point font is used for images. However, there is a great deal of white space within words printed in 14-point font. When solid lines with a thickness greater than the height of 14-point font are substituted for the text in the images with 14point font, the color effect becomes transient. As the solid lines get bigger and bigger, the color effect disappears.

Substituting pictures for words. Suppose pictures that include large black areas are substituted for the images and lines in the previous examples. The size of the large black areas precludes obtaining a good color effect. Figure 4I shows a house and a tree. Image 1 has a large black area for the front of the house and a smaller black area for the tree. When the three images in Figure 4I are repeatedly presented in "Forward" sequential order, the tree trunk, a relatively small black area on the first image, appears to be in brown. The front of the house, a relatively large black area on the image, does not consistently appear to be brown. The grass and tree leaves on the second image appear in blue. If "Backward" is chosen, the tree trunk becomes blue and the grass and leaves become brown.

\section{Availability}

The computer program described in this article is available free of charge on a CD-ROM from the first author. The program was written in Visual Basic 5 and requires an IBM-compatible microcomputer with Windows 95 or higher. The program has been tested on Pentium (I and II) and Athlon $(2400+)$ microcomputers running Windows 95, Windows 98, and Windows XP. The program requires $64 \mathrm{MB}$ of RAM. An installation program is included with the program. From the "Run" option of the "Start" menu, the user types $X$ :Setup where $X$ is the drive letter for the CD-ROM drive. The installation program installs a small program (approximately $70 \mathrm{~KB}$ ) on the computer hard drive. The small program gets the images described in this article from the CD-ROM. Leaving the .bmp images on the CD-ROM saves a great deal of space (approximately $10 \mathrm{MB}$ ) on the hard drive. When the program is run, the images are loaded from the CD-ROM. After the images have loaded, the Fechner wheel demonstrations run as rapidly as they would run on a system that had the images on the hard drive. Of course, the CD-ROM must be in the CD drive when the program is run.

Directory "ProgFile" contains the Visual Basic files for the program. The user can load these files into Visual Basic 5 or Visual Basic 6 and modify the program. The program does not have to be modified to experiment with new images and "rotate" them. Consider the following example. The files in the main directory of the CD can be copied to a directory on the hard drive of a microcomputer. Then, files Fech1.bmp, Fech2.bmp, Fech3.bmp . . Fech64.bmp can be removed. New .bmp files can be drawn using a program for drawing .bmp files. All the files that are in the directory on the hard drive can be copied to a CD. The portion of the program that rotated the original 64 .bmp files will now rotate the new .bmp files.

\section{REFERENCES}

Boring, E. G. (1950). A history of experimental psychology (2nd ed.). New York: Appleton-Century-Crofts.

Hergenhahn, B. R. (2005). An introduction to the history of psychology (5th ed.). Belmont, CA: Thomson Wadsworth.

SwEEnEy, D. (1998). Experience the Fechner phenomenon. Electronics Now, 69, 49-52.

(Manuscript received August 20, 2004; revision accepted for publication February 7, 2005.) 